\title{
Security Strategies for Microservices-based Application Systems
}

Ramaswamy Chandramouli

This publication is available free of charge from: https://doi.org/10.6028/NIST.SP.800-204

\section{O M P U TER SE C UR I T Y}




\section{Security Strategies for Microservices-based Application Systems}

Ramaswamy Chandramouli

Computer Security Division Information Technology Laboratory

This publication is available free of charge from: https://doi.org/10.6028/NIST.SP.800-204

August 2019

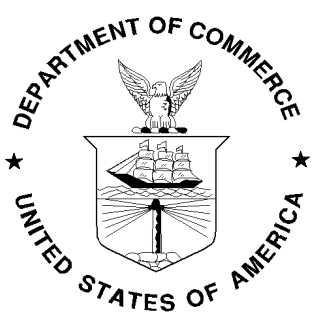

U.S. Department of Commerce Wilbur L. Ross, Jr., Secretary 


\title{
Authority
}

This publication has been developed by NIST in accordance with its statutory responsibilities under the Federal Information Security Modernization Act (FISMA) of 2014, 44 U.S.C. § 3551 et seq., Public Law (P.L.) 113-283. NIST is responsible for developing information security standards and guidelines, including minimum requirements for federal information systems, but such standards and guidelines shall not apply to national security systems without the express approval of appropriate federal officials exercising policy authority over such systems. This guideline is consistent with the requirements of the Office of Management and Budget (OMB) Circular A-130.

Nothing in this publication should be taken to contradict the standards and guidelines made mandatory and binding on federal agencies by the Secretary of Commerce under statutory authority. Nor should these guidelines be interpreted as altering or superseding the existing authorities of the Secretary of Commerce, Director of the OMB, or any other federal official. This publication may be used by nongovernmental organizations on a voluntary basis and is not subject to copyright in the United States. Attribution would, however, be appreciated by NIST.

National Institute of Standards and Technology Special Publication 800-204 Natl. Inst. Stand. Technol. Spec. Publ. 800-204, 50 pages (August 2019) CODEN: NSPUE2

This publication is available free of charge from: https://doi.org/10.6028/NIST.SP.800-204

Certain commercial entities, equipment, or materials may be identified in this document in order to describe an experimental procedure or concept adequately. Such identification is not intended to imply recommendation or endorsement by NIST, nor is it intended to imply that the entities, materials, or equipment are necessarily the best available for the purpose.

There may be references in this publication to other publications currently under development by NIST in accordance with its assigned statutory responsibilities. The information in this publication, including concepts and methodologies, may be used by federal agencies even before the completion of such companion publications. Thus, until each publication is completed, current requirements, guidelines, and procedures, where they exist, remain operative. For planning and transition purposes, federal agencies may wish to closely follow the development of these new publications by NIST.

Organizations are encouraged to review all draft publications during public comment periods and provide feedback to NIST. Many NIST cybersecurity publications, other than the ones noted above, are available at https://csrc.nist.gov/publications.

\section{Comments on this publication may be submitted to:}

\author{
National Institute of Standards and Technology \\ Attn: Computer Security Division, Information Technology Laboratory \\ 100 Bureau Drive (Mail Stop 8930) Gaithersburg, MD 20899-8930 \\ Email: sp800-204-comments@nist.gov
}

\section{All comments are subject to release under the Freedom of Information Act (FOIA).}




\title{
Reports on Computer Systems Technology
}

The Information Technology Laboratory (ITL) at the National Institute of Standards and Technology (NIST) promotes the U.S. economy and public welfare by providing technical leadership for the Nation's measurement and standards infrastructure. ITL develops tests, test methods, reference data, proof of concept implementations, and technical analyses to advance the development and productive use of information technology. ITL's responsibilities include the development of management, administrative, technical, and physical standards and guidelines for the cost-effective security and privacy of other than national security-related information in Federal information systems. The Special Publication 800-series reports on ITL's research, guidelines, and outreach efforts in information system security, and its collaborative activities with industry, government, and academic organizations.

\begin{abstract}
Microservices architecture is increasingly being used to develop application systems since its smaller codebase facilitates faster code development, testing, and deployment as well as optimization of the platform based on the type of microservice, support for independent development teams, and the ability to scale each component independently. Microservices generally communicate with each other using Application Programming Interfaces (APIs), which requires several core features to support complex interactions between a substantial number of components. These core features include authentication and access management, service discovery, secure communication protocols, security monitoring, availability/resiliency improvement techniques (e.g., circuit breakers), load balancing and throttling, integrity assurance techniques during induction of new services, and handling of session persistence. Additionally, the core features could be bundled or packaged into architectural frameworks such as API gateways and service mesh. The purpose of this document is to analyze the multiple implementation options available for each individual core feature and configuration options in architectural frameworks, develop security strategies that counter threats specific to microservices, and enhance the overall security profile of the microservices-based application.
\end{abstract}

\section{Keywords}

microservices; load balancing; circuit breaker; Application Programming Interface (API); API gateway; service mesh; proxy. 


\section{Acknowledgements}

The author, Ramaswamy Chandramouli wishes to thank David Bohannon, Travis Biehn, John Tapp and Jamie Boote from Synopsys and Simon Moffatt of Forgerock for detailed comments on various topics. He also wishes to convey his thanks to Saa Edward Fillie of Wangoh Dynamics Technologies, Carlo de Guzman of DSD Laboratories, and Wei Lien Dang of StackRox. Special thanks to Doug McDorman of T-Mobile for valuable in-line comments and for providing additions to bibliography. Last but not the least, he expresses his thanks to Isabel Van Wyk of G2-Inc for her detailed editorial review.

\section{Patent Disclosure Notice}

NOTICE: NIST's Information Technology Laboratory (ITL) has requested that holders of patent claims whose use may be required for compliance with the guidance or requirements of this publication disclose such patent claims to ITL. However, holders of patents are not obligated to respond to ITL calls for patents and ITL has not undertaken a patent search in order to identify which, if any, patents may apply to this publication.

As of the date of publication and following call(s) for the identification of patent claims whose use may be required for compliance with the guidance or requirements of this publication, no such patent claims have been identified to ITL.

No representation is made or implied by ITL that licenses are not required to avoid patent infringement in the use of this publication. 


\section{Executive Summary}

The microservices paradigm is being increasingly used for designing and deploying large-scale application systems in both cloud-based and enterprise infrastructures. The resulting application system consists of relatively small, loosely coupled entities or components called microservices that communicate with each other using lightweight communication protocols.

Incentives to design and deploy a microservices-based application system include: (a) agility in development due to relatively small and less complex codebases since each one typically implements a single business function; (b) independence among teams in the development process thanks to the loosely coupled nature of microservices; and (c) availability of deployment tools that provide infrastructure services such as authentication, access control, service discovery and communication, and load balancing.

Despite several facilitating technologies (e.g., orchestration), there are many challenges to be addressed in the development and deployment of a microservices-based application. Network security, reliability, and latency are critical factors since every transaction implemented using this type of system will involve the transmission of messages across a network. Further, the presence of multiple microservices exposes a large attack surface.

The goal of this document is to outline strategies for the secure deployment of a microservicesbased application by analyzing implementation options for state of practice core features, configuration options for architectural frameworks such as Application Programming Interface (API) gateway and service mesh, and countermeasures for microservices-specific threats. 


\section{Table of Contents}

Executive Summary ............................................................................................ iv

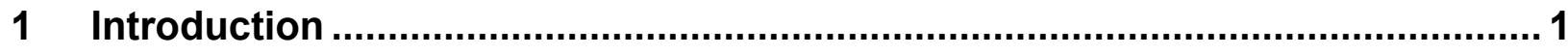

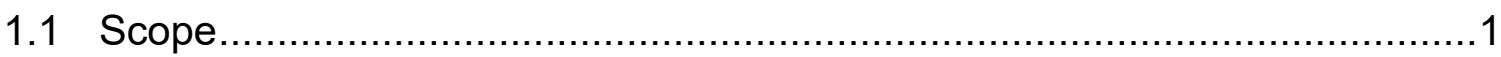

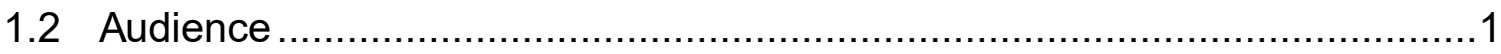

1.3 Relationship to other NIST Guidance Documents ..................................1

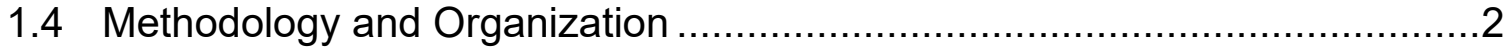

2 Microservices-based Application Systems: Technology Background .............. 3

2.1 Microservices: A Conceptual View.......................................................

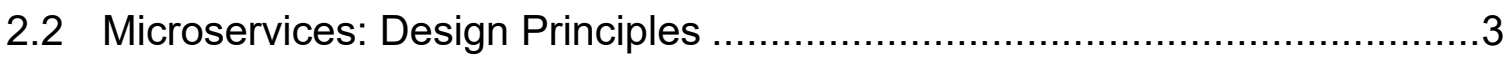

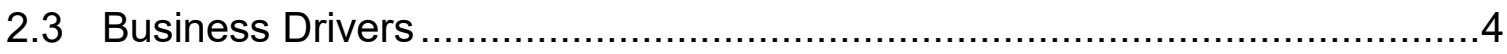

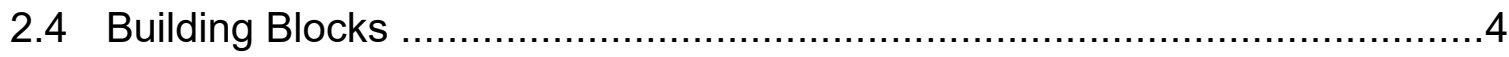

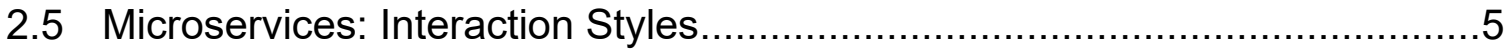

2.6 Microservices: State of the Practice Core Features ...................................

2.7 Microservices: Architectural Frameworks ...............................................

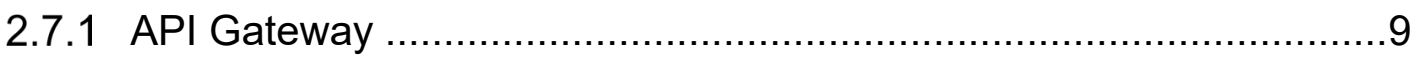

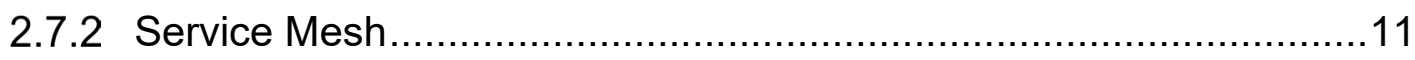

2.8 Comparison with Monolithic Architecture ............................................11

2.9 Comparison with Service-Oriented Architecture (SOA) ….......................12

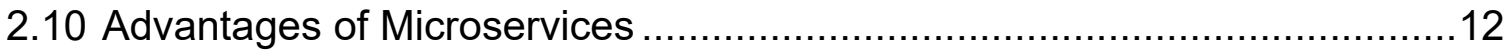

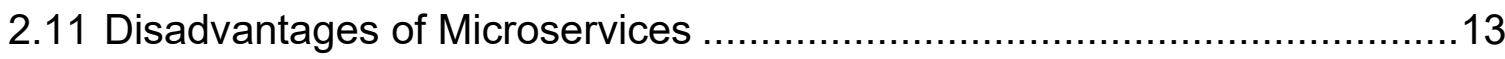

3 Microservices: Threat Background …............................................................ 14

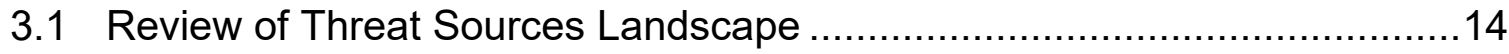

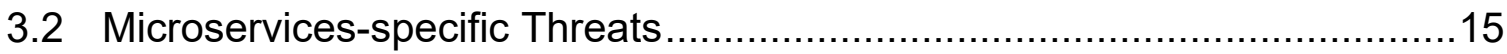

3.2.1 Service Discovery Mechanism Threats .....................................15

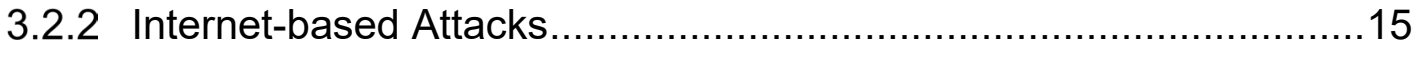

3.2.3 Cascading Failure .............................................................

4 Security Strategies for Implementing Core Features and Countering Threats 17

4.1 Strategies for Identity and Access Management..................................17

4.2 Strategies for Service Discovery Mechanism .......................................19

4.3 Strategies for Secure Communication Protocols …................................21 


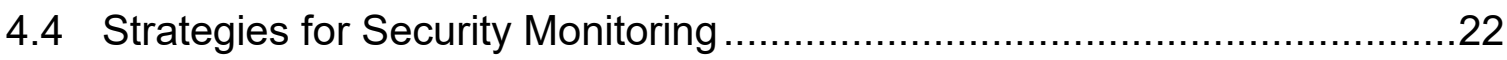

4.5 Availability/Resiliency Improvement Strategies..................................22

4.5.1 Analysis of Circuit Breaker implementation options .......................23

4.5.2 Strategies for Load Balancing ………..................................24

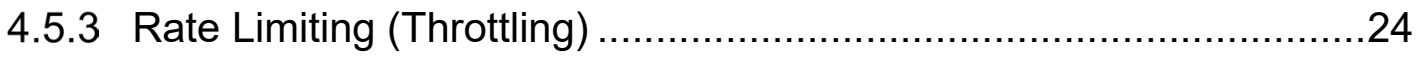

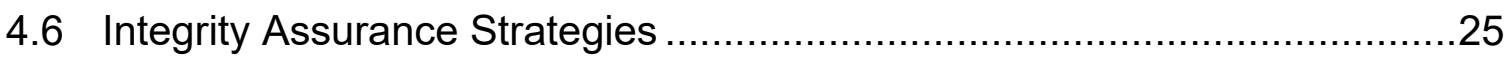

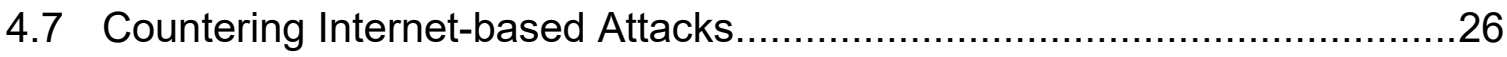

5 Security Strategies for Architectural Frameworks in Microservices ................ 27 Appendix A-Differences between Monolithic Application and Microservices-

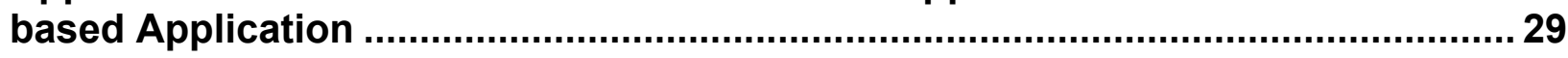

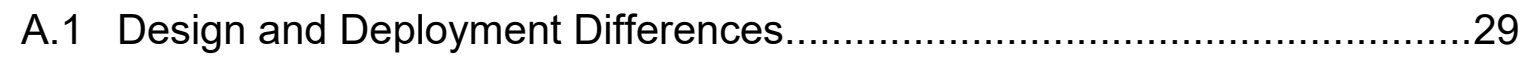

A.1.1 An Example Application to Illustrate the Design and Deployment

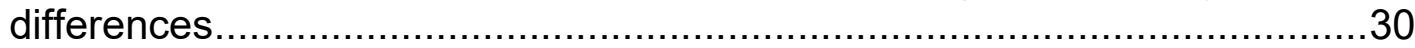

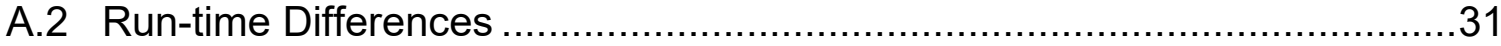

Appendix B-Traceability of Security Strategies to Microservices Architectural

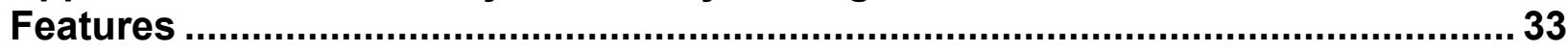

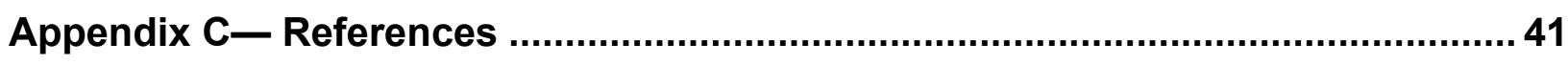




\section{Introduction}

Application systems are increasingly developed and deployed using the microservices paradigm due to advantages such as agility, flexibility, scalability, and availability of tools for automating the underlying processes. However, the tremendous increase in the number of components in a microservices-based application system combined with complex network environments comprised of various interaction styles among components call for several core infrastructure features to be implemented either alone or bundled/packaged into architectural frameworks, such as Application Programming Interface (API) gateway and service mesh. The objective of this document is to perform an analysis of the implementation options for core features, configuration options for architectural frameworks, and countermeasures for microservice-specific threats and outline security strategies.

\subsection{Scope}

This document will not discuss the various tools used in the deployment of microservices-based application systems. Discussion of core features and architectural frameworks will be limited to highlighting issues relevant to secure implementation. The core focus is on the methodology to develop security strategies for microservices-based applications through the following three fundamental steps:

- Study of the technology behind microservices-based application systems focusing on design principles, basic building blocks, and associated infrastructure.

- Focused review of the threat background specific to the operating environment of microservices.

- Analysis of implementation options related to state of practice core features, configuration options related to architectural frameworks such as API gateway and service mesh and countermeasures for microservices-specific threats for developing security strategies.

\subsection{Audience}

The target audience for the security strategies discussed in this document includes:

- Chief Security Officer (CSO) or Chief Technology Officer (CTO) of an IT department in a private enterprise or government agency who wishes to develop enterprise infrastructures to host distributed systems based on microservices architecture; and -Application architects who wish to design a microservices-based application system.

\subsection{Relationship to other NIST Guidance Documents}

This guidance document focuses on a class of application based on a specific architecture. However, since an essential architectural component - the microservice — can be implemented inside a container, the security guidance and recommendations related to application container technology may also serve as relevant security strategies for the application architecture discussed in this document. Such guidance includes: 
- NIST Special Publication (SP) 800-190, Application Container Security Guide; and

- NIST Interagency or Internal Report (NISTIR) 8176, Security Assurance Requirements for Linux Application Container Deployments.

\subsection{Methodology and Organization}

Since microservices-based application systems encompass diverse technologies (e.g., server virtualization, containers, cloud middleware), the focus here is on core features of this application class and the architectural frameworks that bundle or package them. The threat analysis approach involves taking a macro view of the entire deployment stack of microservicesbased application systems and the layer at which these core features are located. The threats specific to those features are identified, and the overall approach for developing security strategies is to analyze the multiple implementations for core features and the architectural frameworks as well as ensure that those implementation options counter microservices-specific threats. The roadmap for the materials used in this methodology is as follows:

- Review of all state of practice core features that form the infrastructure for microservices (Sec. 2.6);

- Review of the layers in the deployment stack, location of the core features in those layers, and identification of microservices-specific threats (Sec. 3);

- Analysis of all different implementation options for these core features and outline security strategies based on these implementation options for core features (Sec. 4); and

- Review of all architectural frameworks that bundle several core features as a single product and outline security strategies based on configuration options for those frameworks (Sec. 5).

A slightly more detailed summarization of the contents of the various sections in this document is as follows:

- Section 2 provides a high-level but expansive overview of microservices-based application systems, starting with a conceptual view followed by design principles, business drivers, building blocks, component interaction styles, state of practice core features, and architectural frameworks;

- Section 3 provides a stack level view of the threat background and some threats that are specific to the microservices environment;

- Section 4 contains analysis information pertaining to various state of practice core features for supporting a microservices-based application and outlines the security strategies for implementing the core features based on analysis of implementation options; and

- Section 5 contains analysis information pertaining to architectural frameworks that bundle core features needed in the infrastructure for microservices-based applications and outlines the security strategies for configuring the architectural frameworks. 


\section{Microservices-based Application Systems: Technology Background}

In this section, the technology behind the development and deployment of a microservices-based application system will be described using the underlying design drivers or principles, the artifacts that constitute the building blocks, and the different ways the building blocks can be configured to produce different deployment options. This is not meant to be a comprehensive description of the technology but provides sufficient information about components and concepts to facilitate the identification of security threats and the development of secure implementation strategies for a microservices-based application system.

\subsection{Microservices: A Conceptual View}

A microservices-based application system consists of multiple components (microservices) that communicate with each other through synchronous remote procedure calls or an asynchronous messaging system. Each microservice typically implements one (rarely more) distinct business process or functionality (e.g., storing customer details, storing and displaying a product catalog, customer order processing etc.). Each microservice is a mini-application that has its own business logic and various adapters for carrying out functions such as database access and messaging. Some microservices would expose a Representational State Transfer (REST)ful API [1] that is consumed by other microservices or by the application's clients [2]. Other microservices might implement a web User Interface (UI). At runtime, a microservice instance may be configured to run as a process in an application server, in a virtual machine (VM), or in a container.

Though a microservices-based application can be implemented purely as an enterprise application and not as a cloud service, it is often identified as a cloud-native application with a service-based architecture, application programming interface (API)-driven communications, container-based infrastructure, and a bias for DevOps (Combination of Development and Operations) processes such as continuous improvement, agile development, continuous delivery, and collaborative development among developers, quality assurance teams, security professionals, IT operations, and line-of-business stakeholders [3]. Part of the reason for this perspective is due to the fact that on-premises software development and deployment relies on a server-centric infrastructure with tightly integrated application modules rather than on loosely coupled, services-based architectures with API-based communications.

\subsection{Microservices: Design Principles}

The design of a microservice is based on the following drivers [4]:

- Each microservice must be managed, replicated, scaled, upgraded, and deployed independently of other microservices.

- Each microservice must have a single function and operate in a bounded context (i.e., have limited responsibility and dependence on other services).

- All microservices should be designed for constant failure and recovery and must therefore be as stateless as possible. 
- One should reuse existing trusted services (e.g., databases, caches, directories) for state management.

These drivers, in turn, result in the following design principles for a microservice:

- Autonomy,

- Loose coupling,

- Re-use,

- Composability,

- Fault tolerance,

- Discoverability, and

- APIs alignment with business processes.

\subsection{Business Drivers}

Though the business drivers for deployment of microservices-based application systems are only marginally related to the theme of this document, it is useful to identify and state those that are relevant from the point of view of user and organizational behavior [5]:

- Ubiquitous access: users want access to applications from multiple client devices (e.g., browsers, mobile devices).

- Scalability: applications must be highly scalable to maintain availability in the face of an increasing number of users and/or increased rate of usage from the existing user base.

- Agile development: organizations want frequent updates to quickly respond to organizational (process and structural) changes and market demands.

\subsection{Building Blocks}

Microservices-based applications (e.g., distributed enterprise or web applications [1]) are built using an architectural style or design pattern that is not restricted to any specific technology and is comprised of small independent entities (end points) that communicate with each other using lightweight mechanisms. These end points are implemented using well-defined APIs. There are several types of API endpoints, such as Simple Object Access Protocol (SOAP) or REST ( Hypertext Transfer Protocol (HTTP) protocol). Each of the small independent entities provides a distinct business capability called a "service" and may have its own data store or repository. Access to these services is provided by various platforms or client types, such as web browsers or mobile devices, using a component called the "client." Together, the component services and the client form the complete microservices-based application system. The services in such a system may be classified as:

- Application-functionality services.

- Infrastructure services (called "core features" in this document) implemented either as stand-alone features or bundled into architectural frameworks (e.g., API gateway, service mesh). These include, but are not limited to, authentication and authorization, service registration and discovery, and security monitoring. 
In a microservices-based application system, each of the multiple, collaborative services can be built using different technologies. This promotes the concept of technical heterogeneity, which means that each service in a microservices-based application system may be written in a different programming language, development platform, or using different data storage technologies. This concept enables developers to choose the right tool or language depending on the type of service. Thus, in a single microservices-based application system, the constituting services may be built using different languages (e.g., Ruby, Golang, Java) and may be hosting different stores (e.g., document datastore, graphical Database (DB), or multimedia DB). Each component service is developed by a team-a microservice or DevOps team - which provides all of the development and operational requirements for that service with a high degree of autonomy regarding development and deployment techniques so long as the service functionality or service contract is agreed upon [6].

Services in microservices are separately deployed on different nodes. The communication between them is transformed from a local function call to a remote call, which could affect system performance due to inherent latency in network communication. Thus, a lightweight communication infrastructure is required.

Scaling can be applied selectively on those services that have performance bottlenecks due to insufficient Central Processing Unit (CPU) or memory resources, while other services can continue to be run using smaller, less expensive hardware. The functionality associated with such a service may be consumed in different ways for different purposes, thereby promoting reusability and composability. One example includes a customer database service, the contents of which are used both by shipping departments for preparing bills of lading and by accounts receivable or the billing department to send invoices.

\subsection{Microservices: Interaction Styles}

In monolithic applications, each component (i.e., a procedure or function) invokes another using a language-level call, such as a method or function. In microservices-based applications, each service is typically a process running in its own distinct network node that communicates with other services through an inter-process communication mechanism (IPC) [7]. Additionally, a service is defined using an interface definition language (IDL) (e.g., Swagger/OpenAPI), resulting in an artifact called the application programming interface (API). The first step in the development of a service involves writing the interface definition, which is reviewed with client developers and iterated multiple times before the implementation of the service begins. Thus, an API serves as a contract between clients and services.

The choice of the IPC mechanism dictates the nature of the API [7]. Table 1provides the nature of API definitions for each IPC mechanism. 
Table 1: IPC Mechanisms and API Types

\begin{tabular}{|c|c|}
\hline \multicolumn{1}{|c|}{ IPC Mechanism } & Nature of API Definition \\
\hline $\begin{array}{c}\text { Asynchronous, message-based } \\
\text { (e.g., Advanced Message Queuing } \\
\text { Protocol (AMQP) or Simple (or } \\
\text { Streaming) Text Oriented Messaging } \\
\text { Protocol. (STOMP)) }\end{array}$ \\
\hline $\begin{array}{c}\text { Synchronous request/response } \\
\text { (e.g., HTTP-based REST or Thrift) }\end{array}$ & $\begin{array}{c}\text { Made up of Uniform Resource Locators (URLs) and } \\
\text { request and response formats }\end{array}$ \\
\hline
\end{tabular}

There can be different types of message formats used in IPC communication: text-based and human-readable, such as JavaScript Object Notation (JSON) or Extensible Markup Language (XML), or of a purely machine-readable binary format, such as Apache Avro or Protocol buffers.

The principle of autonomy described earlier may call for each microservice to be a self-contained entity that delivers all of the functions of an application stack. However, for a microservicesbased application that provides multiple business process capabilities (e.g., an online shopping application that provides business processes such as ordering, shipping, and invoicing), a component microservice is always dependent, in some fashion, on another microservice (e.g., data). In the context of our example, the shipping microservice is dependent upon "unfulfilled orders" data in the ordering microservice to perform its function of generating a shipping or bill of lading record. Hence, there is always the need to couple microservices while still retaining autonomy. The various approaches to creating the coupling, which are often dictated by business process and IT infrastructure needs, include interaction patterns, messaging patterns, and consumption modes. In this document, the term "interaction pattern" is used, and the primary interaction patterns are as follows.

Request-reply: Two distinct types of requests include queries for the retrieval of information and commands for a state-changing business function [2]. In the first type, a microservice makes a specific request for information or to take some action and functionally waits for a response. The purpose of the request for information is retrieval for presentation purposes. In the second type, one microservice asks another to take some action involving a state-changing business function (e.g., a customer modifying their personal profile or submitting an order). In the request-reply pattern, there is a strong runtime dependency between the two microservices involved, which manifests in the following two ways:

- One microservice can execute its function only when the other microservice is available.

- The microservice making the request must ensure that the request has been successfully delivered to the target microservice.

Because of the nature of communication in the request-reply protocol, a synchronous communication protocol, such as HTTP, is used. If the microservice is implemented with a REST API, the messages between the microservices become HTTP REST API calls. The REST APIs are often defined using a standardized language, such as RAML (RESTful API Modeling 
Language), which was developed for microservice interface definition and publication. HTTP is a blocking type of communication wherein the client that initiates a request can continue its task only when it receives a response.

Publish-Subscribe: This pattern is used when microservices need to collaborate for the realization of a complex business process or transaction. This is also called a business domain event-driven approach or domain event subscription approach. In this pattern, a microservice registers itself or subscribes to business domain events (e.g., interested in specific information or being able to handle certain requests), which are published to a message broker through an eventbus interface. These microservices are built using event-driven APIs and use asynchronous messaging protocols, such as Message Queuing Telemetry Transport (MQTT), Advanced Message Queuing Protocol (AMQP), and Kafka Messaging, which enable support for notifications and subscriptions. In asynchronous protocols, the message sender does not typically wait for a response but simply sends the message to a message agent (e.g., RabbitMQ queue). One of the use cases for this approach is the propagation of data updates to multiple microservices based on certain events [8].

\subsection{Microservices: State of the Practice Core Features}

The criticality of the communication infrastructure in a microservices-based application environment calls for several sophisticated capabilities to be provided as core features in many deployments. As already stated, many of these features can be implemented either stand-alone or bundled together in architectural frameworks such as an API gateway or service mesh. Even within the API gateway, these features can be implemented through service composition or direct implementation within the code base. These features include but are not limited to authentication, access control, service discovery, load balancing, response caching, applicationaware health checks, and monitoring [2]. A brief description of these features [5] includes:

- Authentication and access control: Authentication and access policy may vary depending on the type of APIs exposed by microservices — some may be public APIs; some may be private APIs; and some may be partner APIs, which are available only for business partners.

- Service Discovery: In legacy distributed systems, there are multiple services configured to operate at designated locations (IP address and port number). In the microservicesbased application, the following scenario exists and calls for a robust service discovery mechanism:

a) There are a substantial number of services and many instances associated with each service with dynamically changing locations.

b) Each of the microservices may be implemented in VMs or as containers, which may be assigned dynamic IP addresses, especially when they are hosted in an Infrastructure as a Service (IAAS) or Software as a Service (SAAS) cloud service.

c) The number of instances associated with a service can vary based on the load fluctuations using features such as autoscaling.

- Security monitoring and analytics - To detect attacks and identify factors for degradation of services (which may impact availability), it is necessary to monitor network traffic into 
and out of microservices with analytics capabilities in addition to routine logging features.

An API gateway or micro-gateway is generally used for implementing the following core features:

- Optimized endpoint: This involves several capabilities.

a) Request and response collapsing: Most business transactions will involve calls to multiple microservices, often in a pre-determined sequence. An API gateway can simplify the situation for clients by exposing an endpoint that will automatically make all the needed multiple requests (calls) and return a single, aggregated response to the client.

b) API Transformation: The API gateway can provide a public interface to the client which is different from the individual APIs it has to call to cater to a given request. This feature is called API transformation and enables:

i) Changing the implementation and even the API interface for individual microservices; and

ii) Transitioning from an initial, monolithic application to a microservices-based application by enabling continued access to clients through the API gateway while progressively splitting the monolithic application, creating microservice APIs in the background, and changing the API transformation configuration accordingly.

c) Protocol Translation: Calls from clients to microservices entry points may be in web protocols, such as Hypertext Transfer Protocol Secure (HTTPS), while microservices communicate among themselves using synchronous protocols, such as Remote Procedure Call (RPC)/Thrift, or asynchronous protocols, such as AMQP. The necessary protocol translation in client requests is typically carried out by the API gateway.

- Circuit breaker: This is a feature to set a threshold for the failed responses to an instance of a microservice and cut off proxying requests to that instance when the failure is above the threshold. This avoids the possibility of a cascaded failure, allows time to analyze logs, implement the necessary fix, and push an update for the failing instance.

- Load balancing: There is a need to have multiple instances of the same service, and the load on these instances must be evenly distributed to avoid delayed responses or service crashes due to overload.

- Rate limiting (throttling): The rate of requests coming into a microservice must be limited to ensured continued availability of service for all clients.

- Blue/green deployments: When a new version of a microservice is deployed, requests from customers using the old version can be redirected to the new version since the API gateway can be programmed to be aware of the locations of both versions.

- Canary releases: Only a limited amount of traffic is initially sent to a new version of a microservice since the correctness of its response or performance metric under all 
operating scenarios is not fully known. Once sufficient data is gathered about its operating characteristics, then all of the requests can be proxied to the new version of the microservice.

\subsection{Microservices: Architectural Frameworks}

The two main architectural frameworks for bundling or packaging core features that primarily ensure reliable, resilient, and secure communication in a microservices-based application are:

- API gateway, augmented with or without micro gateways; and

- Service mesh.

The role of these frameworks in the operating environment of a microservices-based application system are given in Table 2 [4]:

Table 2: Role of Architectural Frameworks in Microservices Operations

\begin{tabular}{|c|c|}
\hline Architectural Framework & Role in the Overall Architecture \\
\hline $\begin{array}{l}\text { API gateway, augmented with or without } \\
\text { micro gateways }\end{array}$ & Used for controlling north-south and east-west traffic \\
\hline Service mesh & $\begin{array}{l}\text { Deployed for purely east-west traffic when } \\
\text { microservices are implemented using containers but } \\
\text { can also be used in situations where microservices } \\
\text { are housed in VMs or application servers. }\end{array}$ \\
\hline
\end{tabular}

Typical functions in both architectural frameworks include: service discovery, load balancing, failure detection, failure response, and attack monitoring.

\subsubsection{API Gateway}

The API gateway is a popular architectural framework for microservices-based application systems. Unlike a monolithic application where the endpoint may be a single server, a microservices-based application consists of multiple fine-grained endpoints. Hence, it makes sense to provide a single entry point for all clients to multiple component microservices of the application. Another situation where an API gateway is deployed is to act as a front-end (that matches the legacy enterprise software) to back-end services when an organization is migrating from a monolithic enterprise application by gradually replacing its components with independent microservices over time. Direct communication of clients to multiple endpoints results in too many point-to-point connections.

The primary function of the API gateway is to always route inbound requests to the correct down-stream services, optionally perform protocol translation (i.e., translation between web protocols, such as HTTP and WebSocket, and web-unfriendly protocols that are used internally, such as AMQP and Thrift binary RPC) and sometimes compose requests. In some rare instances, they are used as part of a Backend for Frontend (BFF), thus enabling support for clients with different form factors (e.g., browser, mobile device). All requests from clients first go through 
the API gateway, which then routes requests to the appropriate microservice. The API gateway will often handle a request by invoking multiple microservices and aggregating the results.

The multiple APIs or microservices accessible through the API gateway can be specified as part of the input port definition of the gateway (e.g., mobileAPI or MobileService) or be specified dynamically through a deploy operation of the API gateway service with a request parameter that contains the name of the service that should be embedded with the requested service [9]. Thus, the API gateway, located between clients and microservices, represents a pattern wherein a proxy aggregates multiple services. Many API gateway implementations can support APIs written in different languages, such as Jolie, JavaScript, or Java.

Since the API gateway is the entry point for microservices, it should be equipped with the necessary infrastructure services (in addition to its main service of request shaping), such as service discovery, authentication and access control, load balancing, caching, providing custom APIs for each type of client, application-aware health checks, service monitoring, attack detection, attack response, security logging and monitoring and circuit breakers. These additional features may be implemented in the API gateway in two ways:

- By composing the specific services developed for respective functionality (e.g., service registry for service discovery); and

- Implementing these functionalities directly inside the codebase that utilizes the API gateway.

\section{Gateway implementations}

To prevent the gateway from having too much logic to handle request shaping for different client types, it is divided into multiple gateways [8]. This multiple gateway pattern is called BFF. In $\mathrm{BFF}$, each client type is given its own gateway (e.g., web app BFF, mobile app BFF) as a collection point for service requests. The respective backend is closely aligned with the corresponding front end (client) and is typically developed by the same team. The functionality provided by BEF can also be provided by GraphQL which allows the client to shape responses in their requests by specifying what parts of a data-type are required in a response.

API management for a microservices-based application can be implemented through either a monolithic API gateway architecture or a distributed API gateway architecture. In the monolithic API gateway architecture, there is only one API gateway that is typically deployed at the edge of the enterprise network (e.g., Demilitarized Zone (DMZ)) and provides all services to the API at the enterprise level. In the distributed API gateway architecture, there are multiple instances of microgateways, which are deployed closer to microservice APIs [10]. A microgateway is typically a low footprint, scriptable API gateway that can be used to define and enforce customized policies and is therefore suitable for microservices-based applications, which must be protected through service-specific security policies.

The microgateway is typically implemented as a stand-alone container using development platforms such as Node.js. It is different from a sidecar proxy of the service mesh architecture (refer to Section 2.7.2), which is implemented at the API endpoint itself. There are a number of 
ways the security policies can be encoded and input in a gateway. One approach is to encode policies using JSON format and input them through a graphical policy management interface. The microgateway should contain policies for both application requests and responses. When policies and their enforcement are implemented as a container, they are immutable and thus provide a degree of protection against accidental and unintended modifications which may result in security breaches or conflicts. In other words, these types of modifications are prevented when the microgateway is implemented as a container since any security policy update will require redeployment of the microgateway. It is essential that the microgateway deployed for any microservice instance communicate with service registry and monitoring modules to keep track of the operational status of the microservice it is designed to protect.

\subsubsection{Service Mesh}

A service mesh is a dedicated infrastructure layer that facilitates service-to-service communication through service discovery, routing and internal load balancing, traffic configuration, encryption, authentication and authorization, metrics, and monitoring. It provides the capability to declaratively define network behavior, node identity, and traffic flow through policy in an environment of changing network topology due to service instances coming and going offline and continuously being relocated. It can be looked upon as a networking model that sits at a layer of abstraction above the transport layer of the Open System Interconnection (OSI) model (e.g., Transport Control Protocol/Internet Protocol (TCP/IP)) and addresses the service's session layer (Layer 5 of the OSI model) concerns [11]. However, fine-grained authorization may still need to be performed at the microservice since that is the only entity that has the full knowledge of the business logic. A service mesh conceptually has two modules - the data plane and the control plane. The data plane carries the application request traffic between service instances through service-specific proxies. The control plane configures the data plane, provides a point of aggregation for telemetry, and provides APIs for modifying the behavior of the network through various features, such as load balancing, circuit breaking, or rate limiting.

Service meshes create a small proxy server instance for each service within a microservices application. This specialized proxy car is sometimes called a "sidecar proxy" in service mesh parlance [12]. The sidecar proxy forms the data plane, while the runtime operations needed for enforcing security (access control, communication-related) are enabled by injecting policies (e.g., access control policies) into the sidecar proxy from the control plane. This also provides the flexibility to dynamically change policies without modifying the microservices code.

\subsection{Comparison with Monolithic Architecture}

To fully compare the microservice architecture with the monolithic architecture used for all legacy applications, it is necessary to compare the features of applications developed using these architectural styles as well as provide an example of an application under both architectures for a specific business process. A detailed discussion involving these aspects is provided in Appendix A. 


\subsection{Comparison with Service-Oriented Architecture (SOA)}

The architectural style of microservices shares many similarities with service-oriented architecture (SOA) due to the following common technical concepts [13]:

- Services: The application system provides its various functionalities through selfcontained entities or artifacts called services that may have other attributes such as being visible or discoverable, stateless, reusable, composable, or have technological-diversity.

- Interoperability: A service can call any other service using artifacts such as an enterprise service bus (ESB) in the case of SOA or through a remote procedural call (RPC) across a network as in the case of a microservices environment.

- Loose coupling: There is minimal dependency between services such that the change in one service does not require a change in another service.

In spite of the three common technical concepts described above, technical opinion on the relationship between an SOA and microservices environment falls along the following three lines [13]:

- Microservices are a separate architectural style,

- Microservices represent one SOA pattern, and

- A microservice is a refined SOA.

The most prevalent opinion is that the differences between SOA and microservices do not concern the architectural style except in its concrete realization, such as development or deployment paradigms and technologies [2].

\subsection{Advantages of Microservices}

- For large applications, splitting the application into loosely coupled components enables independence between the developer teams assigned to each component. Each team can then optimize by choosing its own development platform, tools, language, middleware, and hardware based on their appropriateness for the component being developed.

- Each of the components can be scaled independently. The targeted allocation of resources results in maximum utilization of resources.

- If components have HTTP RESTful interfaces, implementation can be changed without disruption to the overall function of the application as long as the interface remains the same.

- The relatively smaller codebase involved in each component enables the development team to produce updates more quickly and provide the application with the agility to respond to changes in business processes or market conditions.

- The loose coupling between the components enables containment of the outage of a microservice such that the impact is restricted to that service without a domino effect on other components or other parts of the application. 
- When components are linked together using an asynchronous event-handling mechanism, the impact of a component's outage is temporary since the required functions will automatically execute when the component begins running again, thus maintaining the overall integrity of the business process.

- By aligning the service definition to business capabilities (or by basing the decomposition logic for the overall application functionality based on business processes or capabilities), the overall architecture of the microservices-based system is aligned with the organizational structure. This promotes an agile response when business processes associated with an organizational unit change and consequently require that associated service to be modified and deployed.

- The independent functional nature of a microservice promotes better reusability of the code across applications.

\subsection{Disadvantages of Microservices}

- Multiple components (microservices) must be monitored instead of one single application. A central console is needed to obtain the status of each component and the overall state of the application. Therefore, an infrastructure must be created with distributed monitoring and centralized viewing capabilities.

- The presence of multiple components creates an availability problem since any component may cease functioning at any time.

- A component may have to call the latest version of another component for some clients and call the previous version of the same component for another set of clients (i.e., version management).

- Running an integration test is more difficult since a test environment is needed wherein all components must be working and communicating with each other.

- When interactions within a microservices-based application are designed as API calls, all the necessary processes required for secure API management must be implemented.

- The microservices architecture can break down the practice of defense in depth. Many architectures have a web server running in a DMZ that is expected to be compromised, then a backend service which the web server talks to, and then finally a database that the backend service talks to. The backend service can act as a more hardened layer between the exposed web server and the sensitive data in the database. The microservice architecture tends to collapse this and now the web server and back end service are broken down into microservices potentially more exposed than in the previous model. This can result in fewer layers of protection between the caller and sensitive data. Hence it is critical to securely design and implement the microservices themselves as well as the service mesh or API gateway deployment model. 


\section{Microservices: Threat Background}

The threat background for a microservices-based application system should be treated as a continuation of the technology background provided in Section 2. The following approach has been adopted to review the threat background:

- Consider all layers in the deployment stack of a typical microservices-based application and identify typical potential threats at each layer.

- Identify the distinct set of threats exclusive to microservices-based application systems.

\subsection{Review of Threat Sources Landscape}

Six layers are present in the deployment stack of a typical microservices-based application as suggested in [13]: hardware, virtualization, cloud, communication, service/application, and orchestration. This document considers these layers to be threat sources, and several of the security concerns affiliated with them are described below to provide an overview of the threat background in a microservices-based application. It is important to remember that many of the possible threats are common to other application environments and not specific to a microservices-based application environment.

- Hardware layer: Though hardware flaws, such as Meltdown and Spectre [8], have been reported, such threats are rare. In the context of this document, hardware is assumed to be trusted, and threats from this layer are not considered.

- Virtualization layer: In this layer, threats to microservices or hosting containers originate from compromised hypervisors and the use of malicious or vulnerable container images and VM images. These threats are addressed in other NIST documents and are therefore not discussed here.

- Cloud environment: Since virtualization is the predominant technology used by cloud providers, the same set of threats to the virtualization layer applies. Further, there are potential threats within the networking infrastructure of the cloud provider. For example, hosting all microservices within a single cloud provider may result in fewer networklevel security controls for inter-process communication as opposed to controls for communication between external clients and the microservices hosted within the cloud. Security threats within a cloud infrastructure are considered in several other NIST documents and are therefore not addressed here.

- Communication layer: This layer is unique to microservices-based applications due to the sheer number of microservices, adopted design paradigms (loose coupling and API composition), and different interaction styles (synchronous or asynchronous) among them. Many of the core features of microservices pertain to this layer, and the threats to these core features are identified under microservices-specific threats in Sec. 3.2.

- Service/application layer: In this layer, threats are the results of malicious or faulty code. As this falls under secure application development methodologies, it is outside of the scope of this document. 
- Orchestration layer: An orchestration layer may come into play if the microservices implementation involves technologies such as containers. The threats in this layer pertain to the subversion of automation or configuration features, especially related to scheduling and clustering of servers, containers, or VMs hosting the services, and are therefore beyond the scope of this document.

\subsection{Microservices-specific Threats}

Most state-of-practice core features refer to the communication layer in the deployment stack of microservices-based applications. Hence, the overall security strategies for microservices-based applications should involve choosing the right implementation options, identifying the architectural frameworks packaging those core features, identifying microservice-specific threats, and providing coverage for countering those threats in the implementation options.

However, it should be pointed out that microservices-based applications are still susceptible to most attacks that web applications are susceptible to, including injection, encoding and serialization attacks, cross site scripting (XSS), Cross-Site Request Forgery (CSRF), and HTTP verb tempering [20]. And many of the controls to prevent these attacks still need to be implemented in the microservice code so you need to ensure that developers are not under the impression that an API gateway or service mesh will provide all security for their microservice.

\subsubsection{Service Discovery Mechanism Threats}

The basic functions in a service discovery mechanism are:

- Service registration and de-registration.

- Service discovery.

The potential security threats to the service discovery mechanism include:

- Registering malicious nodes within the system, redirecting communication to them, and subsequently compromising service discovery.

- Corruption of the service registry database leading to redirection of service requests to wrong services and resulting in denial of services; also, redirection to malicious services resulting in compromise of the entire application system.

\subsubsection{Internet-based Attacks}

Though all networked or distributed applications are vulnerable to internet-based attacks, microservices-based applications are more vulnerable to this type of attacks due to the following:

- Unlike a monolithic application that exposes a smaller set of IP-addressable remote procedure call interfaces, a microservices architecture will almost always expose a larger set of IP-addressable remote procedure call interfaces. This is due to the fact that monolithic applications favor single-component implementation of a range of business functions and typically expose a consolidated interface to them all. Applications 
employing a microservices architecture feature many smaller components that coordinate or connect over many interfaces.

- Microservices-based applications have increased risks due to inadvertent exposure of internal functionality, when security controls implemented for upstream components are skipped by directly accessing downstream components. The overall increased complexity of the system increases the chances a developer may omit a check because they can't reason about what conditions the caller has been subjected to.

This class of attacks includes botnet attacks. Though not the only means or being the only class of systems subject to botnet attacks, damage to microservices-based applications could include credential stuffing/abuse, accounts takeover, page scraping and harvesting data, and distributed denial of service.

\subsubsection{Cascading Failure}

The presence of multiple components in a microservices-based application enhances the probability of a failure of a service. Though the components are designed to be loosely coupled from the point of view of deployment, there is a logical or functional dependency since many business transactions require the execution of multiple services in sequence to deliver the required outputs. Therefore, if a service that is upstream in the processing logic of a business transaction fails, other services that depend upon it may become unresponsive as well. This phenomenon is known as cascading failure. 


\section{$4 \quad$ Security Strategies for Implementing Core Features and Countering Threats}

Security strategies for the design and deployment of microservices-based application systems will span the following:

Analysis of implementation options for core features: (Some significant core state of practice features are shown in Appendix B).

a) Identity and access management,

b) Service discovery,

c) Secure communication protocols,

d) Security monitoring,

e) Resiliency or availability improvement techniques, and

f) Integrity assurance improvement techniques.

Countering microservices-specific threats:

a) Threats to service discovery mechanism,

b) Internet-based attacks, and

c) Cascading failures.

Note that service discovery is a core feature in microservices, and analysis of the implementation options will also take into consideration threats to service discovery mechanisms. Similarly, implementation options for resiliency or availability improvement will also address the counter measures for cascading failures. As such, there will not be separate security strategies for these items.

\subsection{Strategies for Identity and Access Management}

Since microservices are packaged as APIs, the initial form of authentication to microservices involves the use of API keys (cryptographic). Authentication tokens encoded in Security Assertion Markup Language (SAML) or through OpenID connect under the OAuth 2.0 framework provide an option for enhancing security [14]. For authorization, a centralized architecture for provisioning and enforcement of access policies governing access to all microservices is required due to the sheer number of services, the implementation of services using APIs, and the need for service composition to support real-world business transactions (e.g., customer order processing and shipping). A standardized, platform-neutral method for conveying authorization decisions through a standardized token (e.g., JSON web tokens (JWT), some of which can be OAuth 2.0 access tokens encoded in JSON format [15]) is also required since each of the microservices may be implemented in a different language or platform framework. Policy provisioning and computation of access decisions require the use of an authorization server.

The disadvantage to implementing access control policies at the access point of each microservice is that additional effort is required to ensure that cross-cutting (common) policies applicable to all microservice APIs are implemented uniformly. Any discrepancy in security 
policy implementation among APIs will have security implications for the entire microservicesbased application, although this applies only to coarse grained policies since fine grained policies can only be specified closer to the microservice or in the microservice itself. Further, the footprint for implementing access control in each microservices node can result in performance issues in some nodes. Since multiple microservices nodes collaborate to perform a transaction, performance problems associated with any node can quickly cascade across multiple services. Taking into consideration these requirements, the strategies for secure identity and access management to microservices are outlined below.

\section{Security strategies for authentication (MS-SS-1):}

- Authentication to microservices APIs that have access to sensitive data should not be done simply by using API keys. Access to such APIs should require authentication tokens that have either been digitally signed (e.g., client credentials grant) or is verified with an authoritative source. Additionally, some services may require either single-use tokens or short-lived tokens (tokens that expire after a short time period) to limit the damage a compromised token can cause.

- Authentication tokens should be handle-based (where initially a token reference is sent to Relying party (RP)), cryptographically signed, or protected by an Hash-based Method Authentication Code (HMAC) scheme.

- Every API Key that is used in the application should have restrictions specified both for the applications (e.g., mobile app, IP address) and the set of APIs where they can be used.

- The restriction scope for functionality of every API Key should be commensurate with the level of assurance provided during identity proofing, whether it be machine or human driven identity proofing.

- When stateless authentication tokens (e.g., JSON Web Tokens (JWT)) are used by implementing shared libraries associated with a microservice, the following security precautions must be observed: (a) the token expiry times should be as short as possible since they determine the duration of the session and an active session cannot be revoked, and (b) the token secret key must not be a part of the library code; it must be a dynamic variable represented by an environmental variable or specified in an environment data file. The key value should be stored in a data vault solution.

- If standards-based techniques such as OAuth or OpenID connect are implemented, they must be deployed securely [19].

\section{Security strategies for access management (MS-SS-2):}

- Access policies to all APIs and their resources should be defined and provisioned to an access server. Access policies at a coarse level of granularity say "Permit to Call for a given set of addressable functionalities" should be defined and enforced at the initial API gateway while authorizations at the finer level of granularity (e.g., related to domain of the particular microservices' business logic) should be defined and enforced closer to the 
location of the microservices (e.g., at the micro gateway) or sometimes at the microservice itself.

- Caching Mechanism: It may be appropriate to allow microservices to cache policy data; this cache should be only relied upon when an access server is unavailable and should expire after a duration appropriate for the environment/infrastructure.

- The access server should be capable of supporting fine-grained policies.

- Access decisions from the access server should be conveyed to individual and sets of microservices through standardized tokens encoded in a platform-neutral format (e.g., OAuth 2.0 token encoded in JSON format). The token can be either a handle-based token or an assertion bearing token.

- The scope of internal authorization tokens appended by the micro gateway or decision point to each request should be carefully controlled; for example, in a request for transaction, the internal authorization token should be limited in scope to only involve the API endpoints that must be accessed for that transaction.

- The API gateway can be leveraged to centralize enforcement of authentication and access control for all downstream microservices, eliminating the need to provide authentication and access control for each of the individual services. If this design is chosen, any component suitably positioned on the network can make anonymous connections to the services bypassing the API gateway and its protections. Mitigating controls such as mutual authentication should be leveraged to prevent direct, anonymous connections to the services.

\subsection{Strategies for Service Discovery Mechanism}

Microservices may have to be replicated and located anywhere in the enterprise or cloud infrastructure for optimal performance and load balancing reasons. In other words, services could be frequently added or removed and dynamically assigned to any network location. Hence, it is inevitable in a microservices-based application architecture to have a service discovery mechanism, which is typically implemented using the service registry. The service registry service is used by microservices that are coming online to publish their locations in a process called service registration and is also used by microservices seeking to discover registered services. The service registry must therefore be configured with confidentiality, integrity, and availability considerations.

In service-oriented architectures (SOA), service discovery is implemented as part of the centralized enterprise service bus (ESB). However, in microservices architecture-where the business functions are packaged and deployed as services within containers and communicate with each other using API calls - it is necessary to implement a lightweight message bus that can implement all three interaction styles mentioned in Section 2.5. Additionally, alternatives to the ways in which service registry service can be implemented span two dimensions: (a) the way clients access the service registry service and (b) centralized versus distributed service registry. Clients can access the service registry service using two primary methods: client-side discovery pattern and server-side discovery pattern [9]. 


\section{Analysis of the client-side service discovery pattern}

The client-side option consists of building registry-aware clients. The client queries the service registry for the location of all services needed to make requests. It then contacts the target service directly. Though simple, this implementation option for service discovery requires the discovery logic (querying the service registry) to be implemented for each programming language and/or framework that is used for client implementations.

\section{Analysis of the service-side service discovery pattern}

The service-side discovery has two implementations: one pattern delegates the discovery logic to a dedicated router service set at a fixed location, while the other utilizes a server in front of each microservice with the functionality of a dynamic Domain Name System (DNS)-resolver (which works with a Domain Name System Security Extensions (DNSSSEC) authoritative server)). In the dedicated router option, the client makes all service requests to this dedicated router service, which in turn queries the service registry for the location of the client-requested service and forwards that request to the discovered location. This removes the tight coupling between an application service and an infrastructure service such as the service registry service. In the DNS resolver pattern, each microservice completes its own service discovery using its built-in DNS resolver to query the service registry. The DNS resolver maintains a table of available service instances and their endpoint locations (i.e., IP addresses). To keep the table up to date, the asynchronous, nonblocking DNS resolver queries the service registry regularly — perhaps every few seconds-using DNS Service records (e.g., Service Resource Records (SRV RRs)) for service discovery. Since the service discovery function through the DNS resolver runs as a background task, the endpoints (URLs) for all peer microservices are instantly available when a service instance needs to make a request [2].

A good strategy would be to use a combination of the service-side service discovery pattern and the client-side service discovery pattern [9]. The former can be used for providing access to all public APIs, while the latter can allow clients to access all cluster-internal interactions.

\section{Centralized versus distributed service registry}

In a centralized service registry implementation, all services wishing to publish their service register at a single point, and all services seeking these services use the single registry to discover them. The security disadvantage of this pattern is the single point of failure [13]. However, data consistency will not be an issue. In the decentralized service registry, there may be multiple service registry instances, and services can register with any of the instances. In the short term, the disadvantage is that there will be data inconsistency between the various service registries. Eventually, consistency among these various instances of service registry is achieved either through broadcasting from one instance to all others or by propagation from one node to all others via attached data in a process called piggybacking.

Regardless of the pattern used for service discovery, secure deployment of service discovery functions should meet the following service registry configuration requirements. 


\section{$\underline{\text { Security strategies for service registry configuration (MS-SS-3) }}$}

- Service registry capabilities should be provided through servers that are either dedicated or part of a service mesh architecture.

- Service registry services should be in a network that has been configured with certain Quality of Service (QoS) parameters to ensure its availability and resilience.

- Communication between an application service and a service registry should occur through a secure communication protocol such as HTTPS or Transport Layer Security (TLS).

- Service registry should have validation checks to ensure that only legitimate services are performing the registration, refresh operations, and database queries to discover services.

- The bounded context and loose coupling principle for microservices should be observed for the service registration/deregistration functions. In other words, the application service should not have tight coupling with an infrastructure service, such as a service registry service, and service self-registration/deregistration patterns should be avoided. When an application service crashes or is running but unable to handle requests, its inability to perform deregistration affects the integrity of the whole process. Therefore, registration/deregistration of an application service should be enabled using a third-party registration pattern, and the application service should be restricted to querying the service registry for service location information as described under the client-side discovery pattern.

- If a third-party registration pattern is implemented, registration/deregistration should only take place after a health check on the application service is performed.

- Distributed service registry should be deployed for large microservices applications, and care should be taken to maintain data consistency among multiple service registry instances.

\subsection{Strategies for Secure Communication Protocols}

Secure communication between clients and services (north-south traffic) and between services (east-west traffic) is critical for the operation of a microservices-based application.

However, certain strategies for security services - such as authentication or the establishment of secure connections - can be handled at the individual microservices nodes. For example, in the fabric model, each microservice instance has the capability to function as an Secure Sockets Layer (SSL) client and SSL server (i.e., each microservice is an SSL/TLS endpoint). Thus, a secure SSL/TLS connection is possible for interservice or inter-process communication from an overall application perspective. These connections can be created dynamically (i.e., before each interservice request) or be created as a keep-alive connection. In the keep-alive connection scheme, a "service A" creates a connection after a full SSL/TLS handshake - the first time an instance of that service makes a request to an instance of a "service B." However, neither service instances terminate the connection after a response returns for that request from service B. Rather, the same connection is reused in future requests. The advantage of this scheme is that the costly overhead involved in performing the initial SSL/TLS handshake can be avoided during 
each request, and an existing connection can be reused for thousands of following interservice requests. Thus, a permanent secure interservice network connection is available for all instances of requests.

\section{Security strategies for secure communication (MS-SS-4)}

- Clients should not be configured to call target services directly but rather to point to the single gateway URL.

- Client to API gateway as well as Service to Service communication should take place after mutual authentication and be encrypted (e.g., using mutual TLS (mTLS) protocol).

- Frequently interacting services should create keep-alive TLS connections.

\subsection{Strategies for Security Monitoring}

Compared to monitoring a monolithic application which runs in a server (or some replicas for load balancing), a microservices-based system must monitor a large number of services, each running in different servers possibly hosted on heterogeneous application platforms. Further, any meaningful transaction in the system will involve at least two or more services.

\section{Security strategies for security monitoring (MS-SS-5)}

- Security monitoring should be performed at both the gateway and service level to detect, alert and respond to inappropriate behavior, for example a bearer token reuse attack and injection attacks. Further, input validation errors and extra parameters errors, crashes and core dumps must be logged. A class of software that can accomplish this is the Open Webapplication Service Project ((OWASP). AppSensor which could be potentially implemented in the gateway, service mesh and microservice itself.

- A central dashboard displays the status of various services and the network segments that link them. At a minimum, the dashboard should show security parameters such as input validation failures and unexpected parameters that are obvious signs of injection attack attempts.

- A baseline for normal, uncompromised behavior in terms of the outcome of business logic decisions, contact attempts, and other behavior should be created. The placement and capabilities of Intrusion Detection System (IDS) nodes should be such that deviations from this baseline can be detected.

\subsection{Availability/Resiliency Improvement Strategies}

In microservices-based applications, targeted efforts that improve the availability or resiliency of certain critical services are needed to enhance the overall security profile of the application. Some technologies that are commonly deployed include:

- Circuit breaker function,

- Load balancing, and

- Rate limiting (throttling). 


\subsubsection{Analysis of Circuit Breaker implementation options}

A common strategy for preventing or minimizing cascading failures involves the use of circuit breakers, which prohibits the delivery of data to the component (microservice) that is failing beyond a specified threshold. This is also known as the fail fast principle. Since the errant service is quickly taken offline, incidences of cascading failures are minimized while the errant component's logs are analyzed, required fixes are performed, and microservices are updated. There are three options for deploying circuit breakers [9]: directly inside the client, on the side of services, or in proxies that operate between clients and services.

Client-side circuit breaker option: In this option, each client has a separate circuit breaker for each external service that the client calls. When the circuit breaker in a client has decided to cut off calls to a service (called "open state" with respect to that service), no message will be sent to the service, and communication traffic in the network is subsequently reduced. Moreover, the circuit breaker functionality need not be implemented in the microservice, which frees valuable resources for efficient implementation of that service. However, locating the circuit breaker in the client carries two disadvantages from a security point of view. First, a great deal of trust must be placed in the client that the circuit breaker code executes properly. Second, the overall integrity of the operation is at risk since knowledge of the unavailability of the service is very much local to the client, a status that is determined based on the frequency of calls from that client to the service rather than on the combined response status received by all clients against that service.

Server-side circuit breaker option: In this option, an internal circuit breaker in the microservice processes all client invocations and decides whether it should be allowed to invoke the service or not. The security advantages of this option are that clients need not be trusted to implement the circuit breaker function, and since the service has a global picture of the frequency of all invocations from all clients, it can throttle requests to a level which it can conveniently handle (e.g., temporarily lighten the load).

Proxy circuit breaker option: In this option, circuit breakers are deployed in a proxy service, located between clients and microservices, which handles all incoming and outgoing messages. Within this, there may be two options: one proxy for each target microservice or a single proxy for multiple services (usually implemented in API gateway) that includes both client-side circuit breakers and service-side circuit breakers existing within that proxy. The security advantage of this option is that neither the client code nor the services code needs to be modified, which avoids trust and integrity assurance issues associated with both these categories of code as well as the circuit breaker function. This option also provides additional protections such as making clients more resilient to faulty services, and shielding services from cases in which a single client sends too many requests [9], resulting in some type of denial of service to other clients that use that service. 


\section{Security strategies for implementing circuit breakers (MS-SS-6)}

- A proxy circuit breaker option should be deployed to limit the trusted component to the proxy. This avoids the need to place the trust on the clients and microservices (e.g., setting thresholds and cutting off requests based on the set threshold) since they are multiple components.

\subsubsection{Strategies for Load Balancing}

Load balancing is an integral functional module in all microservices-based applications, and its main purpose is to distribute loads to services. A service name is associated with a namespace that supports multiple instances of the same service. In other words, many instances of the same service would use the same namespace [13]. To balance the service load, the load balancer chooses one service instance in the request namespace using an algorithm such as the round-robin algorithm - a circular pattern to assign the request to a service instance.

\section{Security strategies for load balancing (MS-SS-7)}

- All programs supporting the load balancing function should be decoupled from individual service requests. For example, the program that performs health checks on services to determine the load balancing pool should run asynchronously in the background.

- Care must be taken to protect the network connection between the load balancer and the microservice platform.

- When a DNS resolver is deployed in front of a source microservice to provide a table of available target microservice instances, it should work in tandem with the health check program to present a single list to the calling microservice.

\subsubsection{Rate Limiting (Throttling)}

The primary goal of rate limiting is to ensure that a service is not oversubscribed impacting availability. That is, when one client increases the rate of requests, the service continues its response to other clients. This is achieved by setting a limit on how often a client can call a service within a defined window of time. When the limit is exceeded, the client-rather than receiving an application-related response-receives a notification that the allowed rate has been exceeded as well as additional data regarding the limit number and the time at which the limit counter will be reset for the requestor to resume receiving responses. A secondary goal of rate limiting is to mitigate the impact of Denial of Service attacks. Closely related to the concept of rate limiting is quota management or conditional rate limiting where limits are determined based on application requirements rather than infrastructure limitations or requirements.

\section{Security strategies for rate limiting (MS-SS-8)}

- Quotas or limits for application usage should be based on both infrastructure and application-related requirements.

- Limits should be determined based on well-defined API usage plans. 
- For high security microservices, replay detection must be implemented. Based on the risk, this feature can be configured to detect replays $100 \%$ of the time or perform random detection.

\subsection{Integrity Assurance Strategies}

Integrity assurance requirements in the context of microservices-based applications arise under two contexts:

- When new versions of microservices are inducted into the system.

- For supporting session persistence during a transaction.

Monitored induction of new releases: Whenever a newer version of a microservice is released, its induction must be a gradual process since (a) all clients may not be ready to use the new version, and (b) the behavior of the new version for all scenarios and use cases may not meet the business process expectation despite extensive testing. To address this situation, a technique called canary release is often adopted [4]. Under this technique, only a limited number of requests are routed to the new version after it is brought online, and the rest are routed to the existing operational version. After a period of observation provides assurance that the new version meets performance and integrity metrics, all of the requests are routed to the new version.

\section{Security (integrity assurance) strategies for the induction of new versions of microservices (MS-SS-9):}

- The traffic to both the existing version and the new version of the service should be routed through a central node, such as an API gateway, to monitor that the blue/green transition occurs in a controlled manner and to monitor the risk associated with a canary release. Security monitoring should cover nodes hosting both the existing and newer versions.

- Usage monitoring of the existing version should steadily increase traffic to the new version.

- The performance and functional correctness of the new version should be factors in increasing traffic to the new version.

- Client preference for the version (existing or new) should be taken into consideration while designing a canary release technique.

Session persistence: It is critical to send all requests in a client session to the same upstream microservice instance since clients execute a complete transaction through multiple requests to a specific service, and the target of all requests should be to the same upstream service instance in that session. This requirement is called session persistence. A situation that could potentially break this requirement is one wherein the microservice stores its state locally, and the load balancer handling individual requests forwards a request from an in-progress user session to a different microservice server or instance. One of the methods for implementing session persistence is sticky cookie. In this method, there is a mechanism to add a session cookie to the first response from the upstream microservice group to a given client, identifying (in an encoded fashion) the server that generated the response. Subsequent requests from the client include the cookie value, and the same mechanism uses it to route the request to the same upstream server [16]. 


\section{Security (integrity assurance) strategies for handling session persistence (MS-SS-10):}

- The session information for a client must be stored securely.

- The artifact used for conveying the binding server information must be protected.

- Internal authorization tokens must not be provided back to the user, and the user's session tokens must not be passed beyond the gateway for use in policy decisions.

\subsection{Countering Internet-based Attacks}

Though it is impossible to protect against all types of Internet-based attacks including botnets, microservice APIs must be provided with detection and prevention capabilities against credential-stuffing and credential abuse attacks as well as the capability to detect malicious botnets. This is especially critical for those applications where each of the microservices are independently callable and carry their own sets of credentials. Credential abuse attacks can be detected using offline threat analysis or run-time solutions [17]. Detection of botnet attacks is provided by a dedicated bot manager product or as an add-on feature in web application firewalls (WAF).

\section{Security strategies for preventing credential abuse and stuffing attacks (MS-SS-11):}

- A run-time prevention strategy for credential abuse is preferable to an offline strategy. A threshold for a designated time interval from a given location (e.g., IP address) for the number of login attempts should be established; if the threshold is exceeded, preventive measures must be triggered by the authentication/authorization server. This feature must be present when a bearer token is used, to detect its reuse and enforce prevention.

- A credential-stuffing detection solution has the capability to check user logins against the stolen credential database and warn legitimate users that their credentials have been stolen.

- Configure IDS and boundary devices to detect the following: (a) a denial of service attack and raise an alert before the service is no longer accessible, and (b) a distributed network probe.

- Configure service hosts to scan file uploads and the contents of each container's memory and file system for resident malware threats. 


\section{$5 \quad$ Security Strategies for Architectural Frameworks in Microservices}

The two main architectural frameworks considered in this document for microservices-based application systems are the API gateway and service mesh. The primary security considerations in the implementation of the API gateway involve choosing the right platform for hosting it, proper integration and configuration with enterprise-wide authentication and authorization frameworks, and securely leveraging the traffic flowing through it for security monitoring and analysis.

\section{Security strategy for API gateway implementation (MS-SS-12):}

- Integrate the API gateway with an identity management application to provision credentials before activating the API.

- When identity management is invoked through the API gateway, connectors should be provided for integrating with identity providers (IdPs).

- The API gateway should have a connector to an artifact that can generate an access token for the client request (e.g., OAuth 2.0 Authorization Server).

- Securely channel all traffic information to a monitoring and/or analytics application for detecting attacks (e.g., denial of service, malicious actions) and unearthing explanations for degrading performance.

- Distributed gateway deployments (or a combination of initial gateway (that intercepts all client accesses) and microgateways (closer to microservices)) should have a token translation (exchange) service [18] between gateways. The token presented to the initial gateway should have permissions with a broad scope whereas the token presented to inside gateways (or microgateways) should be more narrowly scoped with specific permissions or an entirely different token type that is appropriate for the target microservice platform. This helps to implement the least privilege paradigm.

Implementing a service mesh can help ensure that proper configuration parameters associated with various security policies are defined correctly in the control plane so that the intent of the security policies are met, and the service mesh alone does not introduce new vulnerabilities.

\section{Security strategy for service mesh implementation (MS-SS-13):}

- Provide policy support for designating a specific communication protocol between pairs of services and specifying the traffic load between pairs of services based on application requirements.

- The default configuration should always enable access control policies for all services.

- Avoid configurations that may lead to privilege escalation (e.g., the service role permissions and binding of the service role to service user accounts).

- Service mesh deployments should have configuration capabilities to specify resource usage limits for its components. The absence of this feature creates the potential for these components to impact the resiliency and availability of the overall microservices application. 
- Service mesh deployments should have configuration capabilities to collect and send environment metrics, including request metrics, to a centralized service for monitoring. Policies should allow for specifying either a single service mesh or multiple service meshes (each with their own control plane) for multi-cluster microservices environments to ensure high availability and resiliency in those scenarios.

- For highly sensitive microservices-based applications, Layer 3 network segmentation must be configured within the orchestrator platform to complement the Layer 5 network segmentation achieved throughout the service mesh layer. This is a countermeasure to the threat by malicious actors circumventing or bypassing the sidecar proxy that the service mesh uses for firewalling and blocking network traffic. 


\section{Appendix A-Differences between Monolithic Application and Microservices-based Application}

\section{A.1 Design and Deployment Differences}

Conceptually, a monolithic architecture of an application involves generating one huge artifact that must be deployed in its entirety, while a microservices-based application contains multiple self-contained, loosely-coupled executables called services or microservices. The individual services can be deployed independently. In monolithic applications, any change to a certain functionality of the overall application will involve recompilation and, in some instances, retesting of the whole application before being deployed again. However, in the case of microservices, only the relevant service is modified and redeployed since the independent nature of the services ensures that a change in one does not logically affect the functionality of another. In monolithic applications, any increase in workload due to an increase in the number of users or the frequency of application usage will involve allocating resources to the whole application, whereas in microservices, the increase in resources can be selectively applied to those services whose performance is less than desirable, thus providing flexibility in scalability efforts.

Some monolithic applications may be constructed modularly but may not have semantic or logical modularity. Modular construction refers to how an application may be built from a large number of components and libraries that may have been supplied by different vendors, and some components (e.g., database) may be distributed across the network [13]. In such monolithic applications, the design and specification of APIs may be similar to that in a microservices architecture. However, the difference between such modularly designed monolithic applications (sometimes called a classic modular design) and a microservices-based application is that in the latter, the individual API is network-exposed and therefore independently callable and re-usable.

The differences between monolithic and microservices-based applications is summarized in Table 3.

Table 3: Logical Differences between Monolithic and Microservices-based Application

\begin{tabular}{|c|c|}
\hline Monolithic Application & Microservices-based Application \\
\hline Must be deployed as a whole. & $\begin{array}{l}\text { Independent or selective deployment of } \\
\text { services. }\end{array}$ \\
\hline $\begin{array}{l}\text { Change in a small part of the application } \\
\text { requires re-deployment of the entire } \\
\text { application. }\end{array}$ & $\begin{array}{l}\text { Only the modified services need to be re- } \\
\text { deployed. }\end{array}$ \\
\hline $\begin{array}{l}\text { Scalability involves allocating resources } \\
\text { to the application as a whole. }\end{array}$ & $\begin{array}{l}\text { Each of the individual services can be } \\
\text { selectively scaled up by allocating more } \\
\text { resources. }\end{array}$ \\
\hline API calls are local. & $\begin{array}{l}\text { Network-exposed APIs enable } \\
\text { independent invocation and re-usability. }\end{array}$ \\
\hline
\end{tabular}




\section{A.1.1 An Example Application to Illustrate the Design and Deployment differences}

The following example of a small, Online Shopping Application illustrates the design and deployment differences discussed above. The main functions of this application are:

- A module that displays the catalog of products offered by the retailer with pictures of the products, product numbers, product names, and the unit prices;

- A module for processing customer orders by gathering information about the customer (e.g., name, address) and the details of the order (e.g., name of the product from the catalog, quantity, unit price) as well as creating a bin containing all the items ordered in that session;

- A module for preparing the order for shipping, specifying the total bill of lading (i.e., the total package to be shipped, quantity of each item in the order, shipping preferences, shipping address); and

- A module for invoicing the customer with a built-in feature for making payments by credit card or bank account.

The differences in the design of this Online Shopping Application as a monolithic versus microservices-based are given in Table 4. Schematic diagrams of this application under monolithic and microservices architectures are given in Figures 1 and 2 respectively.

Table 4: Differences in Application Construct between Monolithic and Microservices-based Application

\begin{tabular}{|c|c|c|}
\hline Application Construct & Monolith & Microservices-based \\
\hline $\begin{array}{l}\text { Communication between } \\
\text { functional modules }\end{array}$ & $\begin{array}{l}\text { All communications are in the } \\
\text { form of procedure calls or } \\
\text { some internal data structures } \\
\text { (e.g., socket). The module } \\
\text { handling the order } \\
\text { processing makes a } \\
\text { procedural call to the module } \\
\text { handling the shipping } \\
\text { function and waits for } \\
\text { successful completion } \\
\text { (blocking type synchronous } \\
\text { communication). }\end{array}$ & $\begin{array}{l}\text { The shipping functionality } \\
\text { and the order processing } \\
\text { functionality are each } \\
\text { designed as independent } \\
\text { services. Communication } \\
\text { takes place as an API call } \\
\text { across the network using a } \\
\text { web protocol. The order } \\
\text { processing microservice can } \\
\text { either (a) make a request- } \\
\text { response call to the shipping } \\
\text { microservice and wait for a } \\
\text { response or (b) put the } \\
\text { details of the order to be } \\
\text { shipped in a message queue } \\
\text { to be picked up } \\
\text { asynchronously by the } \\
\text { shipping microservice, which } \\
\text { has subscribed to the event. }\end{array}$ \\
\hline $\begin{array}{l}\text { Handling changes or } \\
\text { enhancements (e.g., } \\
\text { invoicing module needs to be } \\
\text { changed to accept debit } \\
\text { cards) }\end{array}$ & $\begin{array}{l}\text { The entire application must } \\
\text { be recompiled and } \\
\text { redeployed after making the } \\
\text { necessary changes. }\end{array}$ & $\begin{array}{l}\text { The invoicing function is } \\
\text { designed as a separate } \\
\text { microservice, so that service } \\
\text { can simply be recompiled } \\
\text { and redeployed. }\end{array}$ \\
\hline
\end{tabular}




\begin{tabular}{|c|c|c|}
\hline Application Construct & Monolith & Microservices-based \\
\hline $\begin{array}{l}\text { Scaling the application, } \\
\text { allocation of increased } \\
\text { resources (e.g., order } \\
\text { processing module needs to } \\
\text { be allocated more resources } \\
\text { to handle a larger load) }\end{array}$ & $\begin{array}{l}\text { The order processing } \\
\text { functionality involves longer } \\
\text { transaction times compared } \\
\text { to shipping or invoicing } \\
\text { functions. Vertical scaling } \\
\text { that involves using servers } \\
\text { with more memory or CPUs } \\
\text { must be deployed for the } \\
\text { entire application. }\end{array}$ & $\begin{array}{l}\text { It is enough to allocate } \\
\text { increased resources for } \\
\text { hardware where the order } \\
\text { processing microservice is } \\
\text { deployed. Also, the number } \\
\text { of instances of order- } \\
\text { processing microservices } \\
\text { can be increased for better } \\
\text { load balancing. }\end{array}$ \\
\hline $\begin{array}{l}\text { Development and } \\
\text { deployment strategy }\end{array}$ & $\begin{array}{l}\text { Development is handled by } \\
\text { the development team which, } \\
\text { after necessary testing by the } \\
\text { QA team, transfers the task } \\
\text { of deployment to an } \\
\text { infrastructure team that } \\
\text { oversees the allocation of } \\
\text { suitable resources for } \\
\text { deployment. }\end{array}$ & $\begin{array}{l}\text { The complete lifecycle-from } \\
\text { development to } \\
\text { deployment-is handled by a } \\
\text { single DevOps team for each } \\
\text { microservice since it is a } \\
\text { relatively small module with a } \\
\text { single functionality and built- } \\
\text { in platform (e.g., OS, } \\
\text { languages libraries) that is } \\
\text { optimal for that functionality. }\end{array}$ \\
\hline
\end{tabular}

\section{A.2}

\section{Run-time Differences}

A monolithic application runs as a single computational node such that the node is aware of the overall system or application state. In a microservices environment, the application is designed as a set of multiple nodes that each provide a service. Since they operate without the need to coordinate with others, the overall system state is unknown to individual nodes. In the absence of any global information or global variable values, the individual nodes make decisions based on locally available information. The independence of the nodes means that failure of one node does not affect other nodes. Unlike monolithic applications where services may share database connections or a data repository, a microservice architecture may deploy a pattern wherein each service has its own data repository. In many situations, interaction between services may require a distributed transaction which, if not designed properly, may affect the integrity of the databases.

The runtime differences between monolithic and microservices applications and their implications are summarized in Table 5.

Table 5: Architectural Differences between Monolithic and Microservices-based Application

\begin{tabular}{|c|c|}
\hline Monolithic Application & Microservices-based Application \\
\hline $\begin{array}{l}\text { Runs as a single computational node; overall } \\
\text { state information fully known. }\end{array}$ & $\begin{array}{l}\text { Designed as a set of multiple nodes, each } \\
\text { providing a service; overall system state is } \\
\text { unknown to individual nodes. }\end{array}$ \\
\hline $\begin{array}{l}\text { Designed to make use of global information } \\
\text { or values of global variables. }\end{array}$ & $\begin{array}{l}\text { Individual nodes make decisions based on } \\
\text { locally available information. }\end{array}$ \\
\hline $\begin{array}{l}\text { Failure of the node means crash of the } \\
\text { application. }\end{array}$ & $\begin{array}{l}\text { Failure of one node should not affect other } \\
\text { nodes. }\end{array}$ \\
\hline
\end{tabular}




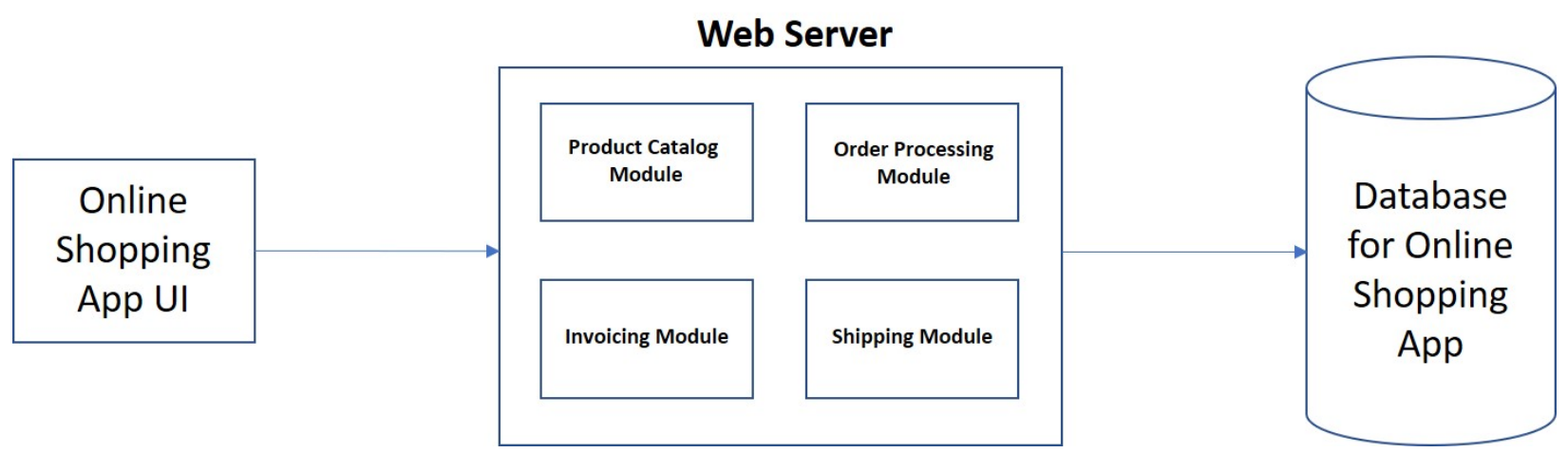

Figure 1: Online Shopping Application - Monolithic Architecture

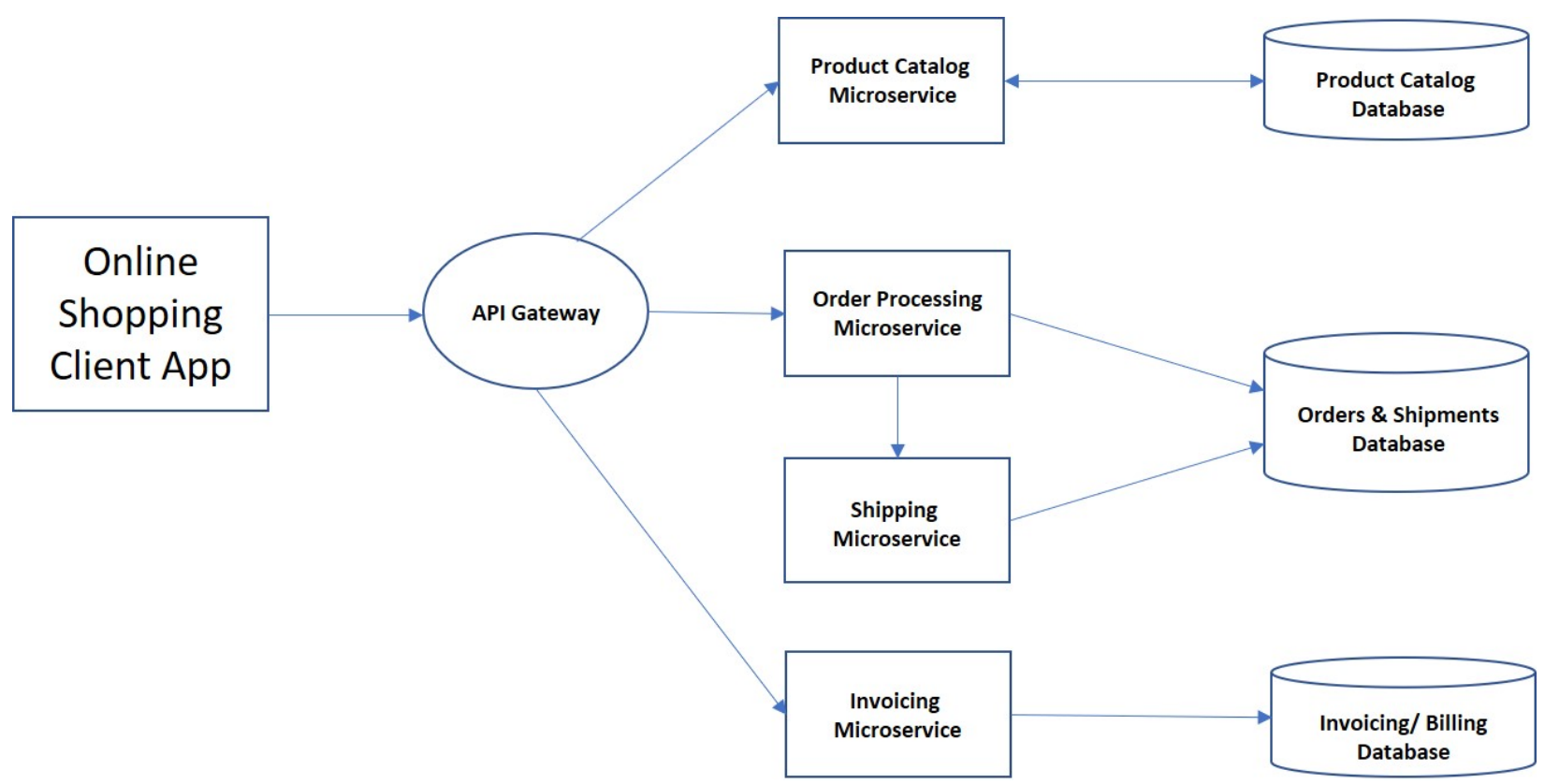

Figure 2: Online Shopping Application - Microservices Architecture 


\section{Appendix B-Traceability of Security Strategies to Microservices Architectural Features}

All security strategies discussed in sections $4 \& 5$ (a total of 13) are listed in Table 6 along with either the microservice core feature or the architectural framework to which each of them pertain. 


\begin{tabular}{|c|c|c|}
\hline $\begin{array}{l}\text { Security Strategy } \\
\text { Identifier }\end{array}$ & Security Strategy & $\begin{array}{l}\text { Microservices Core Feature I } \\
\text { Architectural Framework }\end{array}$ \\
\hline MS-SS-1 & $\begin{array}{l}\text { - Authentication to microservices APIs that have access to sensitive data should } \\
\text { not be done simply by using API keys. Access to such APIs should require } \\
\text { authentication tokens that have either been digitally signed (e.g., client } \\
\text { credentials grant) or that is verified with an authoritative source. Additionally, } \\
\text { some services may require either single-use tokens or short-lived tokens } \\
\text { (tokens that expire after a short time period) to limit the damage a compromised } \\
\text { token can cause. } \\
\text { - Authentication tokens should be handle-based, cryptographically signed, or } \\
\text { protected by an HMAC scheme. } \\
\text { - Every API Key that is used in the application should have restrictions specified } \\
\text { both for the applications (e.g., mobile app, IP address) and the set of APIs } \\
\text { where they can be used. } \\
\text { - The restriction scope for functionality of every API Key should be } \\
\text { commensurate with the level of assurance provided during identity proofing, } \\
\text { whether it be machine or human driven identity proofing. } \\
\text { When stateless authentication tokens (e.g., JSON Web Tokens (JWT)) are } \\
\text { used by implementing shared libraries associated with a microservice, the } \\
\text { following security precautions must be observed: (a) the token expiry times } \\
\text { should be as short as possible since they determine the duration of the session } \\
\text { and an active session cannot be revoked, and (b) the token secret key must not } \\
\text { be a part of the library code, it must be a dynamic variable represented by an } \\
\text { environmental variable or specified in an environment data file. The key value } \\
\text { should be stored in a data vault solution. } \\
\text { If standards-based techniques such as OAuth or OpenID connect are } \\
\text { implemented, they must be deployed securely [19]. }\end{array}$ & Authentication \\
\hline
\end{tabular}


Security Strategy Identifier

\section{Security Strategy}

- Access policies to all APIs and their resources should be defined and provisioned to an access server. Access policies at a coarse level of granularity say "Permit to Call for a given set of addressable functionalities" should be defined and enforced at the initial API gateway while authorizations at the finer level of granularity (e.g., related to domain of the particular microservices' business logic) should be defined and enforced closer to the location of the microservices (e.g., at the microgateway) or sometimes at the microservice itself.

- Caching Mechanism: It may be appropriate to allow microservices to cache policy data; this cache should be only relied upon when an access server is unavailable and should expire after a duration appropriate for the environment/infrastructure.

- The access server should be capable of supporting fine-grained policies.

- Access decisions from the access server should be conveyed to individual and sets of microservices through standardized tokens encoded in a platformneutral format (e.g., OAuth 2.0 token encoded in JSON format). The token can be either a handle-based token or an assertion bearing token.

- The scope of internal authorization tokens appended by the micro gateway or decision point to each request should be carefully controlled; for example, in a request for transaction, the internal authorization token should be limited in scope to only involve the API endpoints that must be accessed for that transaction.

- The API gateway can be leveraged to centralize enforcement of authentication and access control for all downstream microservices, eliminating the need to provide authentication and access control for each of the individual services. If this design is chosen, any component suitably positioned on the network can make anonymous connections to the services bypassing the API gateway and its protections. Mitigating controls such as mutual authentication should be leveraged to prevent direct, anonymous connections to the services."
Microservices Core Feature / Architectural Framework

Access management 


\section{Security Strategy} Identifier

\section{Security Strategy}

Microservices Core Feature I Architectural Framework

Service registry configuration
- Service registry capabilities should be provided through servers that are either dedicated or part of a service mesh architecture.

- Service registry services should be in a network that has been configured with certain QoS parameters to ensure its availability and resilience.

- Communication between an application service and a service registry should be through a secure communication protocol, such as HTTPS/TLS.

- Service registry should have validation checks to ensure that only legitimate services are performing the registration and refresh operations or querying its database to discover services

- The bounded context and loose coupling principle for microservices should be observed for the service registration/deregistration function; the application service should not have tight coupling with an infrastructure service, such as service registry service, and the service self-registration/deregistration pattern should be avoided. Moreover, when an application service crashes or is running but not in a position to handle requests, it cannot perform deregistration, thus affecting the integrity of the whole process. Registration or deregistration of an application service should be enabled using a third-party registration pattern, and the application service should be restricted to simply querying the service registry for service location information as described in the client-side discovery pattern.

- If a third-party registration pattern is implemented, registration/deregistration should only take place after performing a health check on the application service

- Distributed service registry should be deployed for large microservices applications, and care should be taken to maintain data consistency among multiple service registry instances

- Clients should not be configured to call their target services directly but rather be configured to point to the single gateway URL

- Client to API gateway as well as Service to Service communication should take place after mutual authentication and be encrypted (e.g., using mTLS protocol)

- Frequently interacting services should create keep-alive TLS connections 


\begin{tabular}{|c|c|c|}
\hline $\begin{array}{l}\text { Security Strategy } \\
\text { Identifier }\end{array}$ & Security Strategy & $\begin{array}{l}\text { Microservices Core Feature / } \\
\text { Architectural Framework }\end{array}$ \\
\hline MS-SS-5 & $\begin{array}{l}\text { - Security monitoring should be performed at both the gateway and service level } \\
\text { to detect, alert and respond to inappropriate behavior, for example a bearer } \\
\text { token reuse attack and injection attacks. Further, input validation errors and } \\
\text { extra parameters errors, crashes and core dumps must be logged. A class of } \\
\text { software that can accomplish this is the OWASP AppSensor which could } \\
\text { potentially be implemented in the gateway, service mesh and microservice } \\
\text { itself. } \\
\text { - A central dashboard displays the status of various services and the network } \\
\text { segments that link them. At a minimum, the dashboard should show security } \\
\text { parameters such as input validation failures and unexpected parameters that } \\
\text { are obvious signs of injection attack attempts. } \\
\text { A baseline for normal, uncompromised behavior in terms of the outcome of } \\
\text { business logic decisions, contact attempts, and other behavior should be } \\
\text { created. The placement and capabilities of IDS nodes should be such that } \\
\text { deviations from this baseline can be detected. }\end{array}$ & Security monitoring \\
\hline MS-SS-6 & $\begin{array}{l}\text { A proxy circuit breaker option should be deployed to limit the trusted component } \\
\text { to be the proxy, which avoids the need to place the trust on the clients and } \\
\text { microservices (e.g.,setting thresholds and cutting off requests based on the set } \\
\text { threshold) since they are multiple components }\end{array}$ & Implementing circuit breaker \\
\hline MS-SS-7 & $\begin{array}{l}\text { The load balancing function should be decoupled from individual service } \\
\text { requests; for example, the program that performs health checks on the services } \\
\text { to determine the load balancing pool should run asynchronously in the } \\
\text { background } \\
\text { - Care must be taken to protect the network connection between the load } \\
\text { balancer and the microservice platform. } \\
\text { When a DNS resolver is deployed in front of a source microservice to provide a } \\
\text { table of available target microservice instances, it should work in tandem with } \\
\text { the health check program to present a single list to the calling microservice }\end{array}$ & Implementing load balancing \\
\hline
\end{tabular}




\begin{tabular}{|c|c|c|}
\hline $\begin{array}{l}\text { Security Strategy } \\
\text { Identifier }\end{array}$ & Security Strategy & $\begin{array}{c}\text { Microservices Core Feature I } \\
\text { Architectural Framework }\end{array}$ \\
\hline MS-SS-8 & $\begin{array}{l}\text { - Quotas or limits for application usage should be based on both infrastructure } \\
\text { and application-related requirements } \\
\text { - Limits should be determined based on well-defined API usage plans } \\
\text { - For high security microservices, replay detection must be implemented. Based } \\
\text { on the risk, this feature can be configured to detect replays } 100 \% \text { of the time or } \\
\text { perform random detection. }\end{array}$ & Rate limiting (throttling) \\
\hline MS-SS-9 & $\begin{array}{l}\text { - The traffic to both the existing version and the new version of the service should } \\
\text { be routed through a central node, such as an API gateway, to monitor that the } \\
\text { blue/green transition occurs in a controlled manner and to monitor the risk } \\
\text { associated with a canary release. Security monitoring should cover nodes } \\
\text { hosting both the existing and newer versions } \\
\text { - Usage monitoring of the existing version should drive the rate of "ramping up" of } \\
\text { the traffic to the new version. } \\
\text { - The performance and functional correctness of the new version should be a } \\
\text { factor in the ramping up of the traffic to the new version. } \\
\text { - Client preference for the version (existing or new) should be taken into } \\
\text { consideration while designing a canary release technique. }\end{array}$ & $\begin{array}{l}\text { Induction of new versions of } \\
\text { microservice }\end{array}$ \\
\hline MS-SS-10 & $\begin{array}{l}\text { - Session information for a client must be stored securely } \\
\text { - The artifact used for conveying the binding server information must be protected } \\
\text { Internal authorization tokens must not be provided back to the user, and the } \\
\text { user's session tokens must not be passed beyond the gateway for use in policy } \\
\text { decisions. }\end{array}$ & Handling session persistence \\
\hline
\end{tabular}


Security Strategy Identifier

MS-SS-11

\begin{tabular}{|l|l}
\hline MS-SS-11 & \\
\hline MS-SS-12 & $\bullet$ \\
& $\bullet$ \\
$\bullet$ & $\bullet$ \\
$\bullet$
\end{tabular}

\section{Security Strategy}

Microservices Core Feature I Architectural Framework

- A run-time prevention strategy for credential abuse is preferable to an offline strategy. A threshold for a designated time interval from a given location (e.g., IP address) for the number of login attempts should be established; if the threshold is exceeded, preventive measures must be triggered by the authentication/authorization server. This feature must be present when a bearer token is used, to detect its reuse and enforce prevention.

- A credential-stuffing detection solution has the capability to check user logins against the stolen credential database and warn legitimate users that their credentials have been stolen.

- Configure IDS and boundary devices to detect the following: (a) a denial of service attack and raise an alert before the service is no longer accessible, and (b) a distributed network probe.

- Configure service hosts to scan file uploads and the contents of each container's memory and file system for resident malware threats.

- Integrate the API gateway with an identity management application to provision credentials before activating the API.

- When identity management is invoked through the API gateway, connectors should be provided for integrating with identity providers (IdPs).

- The API gateway should have a connector to an artifact that can generate an access token for the client request (e.g., OAuth 2.0 Authorization Server).

- Securely channel all traffic information to a monitoring and/or analytics application for detecting attacks (e.g., denial of service, malicious actions) and unearthing explanations for degrading performance.

- Distributed gateway deployments (or a combination of initial gateway (that intercepts all client accesses) and microgateways (closer to microservices)) should have a token translation (exchange) service [18] between gateways. The token presented to the initial gateway should have permissions with a broad scope whereas the token presented to inside gateways (or microgateways) should be more narrowly scoped with specific permissions or an entirely different token type that is appropriate for the target microservice platform. This helps to implement the least privilege paradigm.
Preventing credential abuse and stuffing attacks

API gateway configuration 
Security Strategy Identifier

\section{Security Strategy}

- Policy support should be enabled for: (a) designating a specific communication protocol between pairs of services and (b) specifying the traffic load between pairs of services based on application requirements

- The default configuration should always be to enable access control policies for all services

- Avoid configurations that may lead to privilege escalation (e.g., the service role permissions and binding of the service role to service user accounts)

- Service mesh deployments should have configuration capabilities to specify resource usage limits for its components. The absence of this feature creates the potential for these components to impact the resiliency and availability of the overall microservices application.

- Service mesh deployments should have configuration capabilities to collect and send environment metrics, including request metrics, to a centralized service for monitoring. Policies should allow for specifying either a single service mesh or multiple service meshes (each with their own control plane) for multi-cluster microservices environments to ensure high availability and resiliency in those scenarios.

- For highly sensitive microservices-based applications, Layer 3 network segmentation must be configured within the orchestrator platform to complement the Layer 5 network segmentation achieved throughout the service mesh layer. This is a countermeasure to the threat by malicious actors circumventing or bypassing the sidecar proxy that the service mesh uses for firewalling and blocking network traffic.
Microservices Core Feature I Architectural Framework

Service mesh configuration 


\section{Appendix C-References}

[1] Sill A (2016) The design and architecture of microservices. IEEE Cloud Computing 3(5):76-80. https://doi.org/10.1109/MCC.2016.111

[2] Richardson C, Smith F (2016) Microservices: From design to deployment (NGINX Inc.). Available at https://www.nginx.com/resources/library/designing-deployingmicroservices/

[3] TechTarget (n.d.) Comparing two schools of application development: Traditional vs. Cloud-Native. Available at https://searchcloudcomputing.techtarget.com/PaaS/Comparing-Two-Schools-ofApplication-Development-Traditional-vs-Cloud-Native

[4] Richardson C (2015) Building microservices: Using an API gateway. Available at https://www.nginx.com/blog/building-microservices-using-an-api-gateway/

[5] Palladino M (2016) Microservices and API gateway, Part 1: Why an API gateway? Available at https://shadrin.org/nginx/blog/content/microservices-api-gateways-part-1why-an-api-gateway.html

[6] Jander K, Braubach L, Pokahr A (2018) Defense in-depth and role authentication for microservice systems. Procedia Computer Science 130:456-463.

https://doi.org/10.1016/j.procs.2018.04.047

[7] Richardson C (2015) Building microservices: Inter-process communication in a microservices architecture. Available at https://www.nginx.com/blog/buildingmicroservices-inter-process-communication/

[8] Harms H, Rogowski C, Lo Iacono L (2017) Guidelines for adopting frontend architectures and patterns in microservices-based systems. Proceedings of the 2017 11th Joint Meeting on Foundations of Software Engineering (ACM, Paderborn, Germany), pp 902-907. https://doi.org/10.1145/3106237.3117775

[9] Montesi F, Weber J (2016) Circuit breakers, discovery, and API gateways in microservices. arXiv preprint. https://arxiv.org/abs/1609.05830v2

[10] O’Neill M, Malinverno P (2018) Critical capabilities for full life cycle API management. (Gartner, Stamford, CT), ID G00334223. Available at https://www.gartner.com/doc/reprints?id=1-51SE2EK\&ct $=180601 \& \mathrm{st}=\mathrm{sb}$

[11] Calcote L (2018) The enterprise path to service mesh architectures (O'Reilly Media, Sebastopol, CA). 
[12] Twistlock (n.d.) Securing the service mesh: Understanding the value of service meshes, why Istio is rising in popularity, and exploring official Twistlock compliance checks for Istio. Available at https://www.twistlock.com/resources/securing-service-mesh-istiocompliance-checks/

[13] Yarygina T, Bagge, AH (2018). Overcoming security challenges in microservice architecture. Proceedings of 2018 IEEE Symposium on Service-Oriented System Engineering (IEEE, Bamberg, Germany), pp 11-20. https://doi.org/10.1109/SOSE.2018.00011

[14] OpenID (2019) Welcome to OpenID Connect. Available at https://openid.net/connect/

[15] Hardt D (ed.) (2012) The OAuth 2.0 authorization framework. (Internet Engineering Task Force), IETF Request for Comments (RFC) 6749. https://doi.org/10.17487/RFC6749

[16] NGINX (n.d.) High-performance load balancing: Scale out your applications with NGINX and NGINX Plus. Available at https://www.nginx.com/products/nginx/load$\underline{\text { balancing/ }}$

[17] Katz O (2017) Improving credential abuse threat mitigation. Available at https://blogs.akamai.com/2017/01/improving-credential-abuse-threat-mitigation.html

[18] Jones M, Nadalin A, Campbell B (ed.), Bradley J, Mortimore C (2018) OAuth 2.0 token exchange. (Internet Engineering Task Force), IETF Internet-Draft. Available at https://datatracker.ietf.org/doc/draft-ietf-oauth-token-exchange/

[19] Lodderstedt T, Bradley J, Labunets A, Fett D (2019) OAuth 2.0 security best current practice. (Internet Engineering Task Force), IETF Internet-Draft. Available at https://datatracker.ietf.org/doc/draft-ietf-oauth-security-topics/

[20] Jain J (2015) HTTP verb tempering: Bypassing web authentication and authorization. Available at https://resources.infosecinstitute.com/http-verb-tempering-bypassing-webauthentication-and-authorization/ 